\title{
DIABETES, OBESITY AND METABOLISM
}

\section{A JOURNAL OF PHARMACOLOGY AND THERAPEUTICS}

\section{Beneficial effects of replacing diet beverages with water on Type 2 diabetic obese women following a hypo-energetic diet - a randomized, 24 week clinical trial}

\begin{tabular}{|r|l|}
\hline Journal: & Diabetes, Obesity and Metabolism \\
\hline Manuscript ID & DOM-16-0384-OP.R1 \\
\hline Danuscript Type: & Original Paper \\
\hline Complete List of Authors: & $\begin{array}{l}\text { Madjd, Ameneh; The University of Nottingham, School of Life Sciences; } \\
\text { Novindiet Clinic } \\
\text { Taylor, Moira; The University of Nottingham, School of Life Sciences } \\
\text { Delavari, Alireza; Tehran University of Medical Sciences Digestive Disease } \\
\text { Research Center } \\
\text { Malekzadeh, Reza; Tehran University of Medical Sciences Digestive Disease } \\
\text { Research Center } \\
\text { MacDonald, Ian; School of Life Sciences, E Floor, Medical School } \\
\text { Farshchi, Hamid; The University of Nottingham, School of Life Sciences; } \\
\text { Novindiet Clinic }\end{array}$ \\
\hline Key Words: & \begin{tabular}{l} 
insulin resistance, type 2 diabetes, clinical trial, obesity therapy \\
\hline
\end{tabular} \\
\hline
\end{tabular}




\section{Title page}

Title of the article:

Beneficial effects of replacing diet beverages with water on Type 2 diabetic obese women following a hypo-energetic diet - a randomized, 24 week clinical trial Ameneh Madjd ${ }^{1,2}$ (D. Pharm), Moira A. Taylor ${ }^{1}$ (PhD), Alireza Delavari ${ }^{3}$ (MD), Reza Malekzadeh $^{3}(\mathrm{MD})$, lan A. Macdonald ${ }^{1}(\mathrm{PhD})$ and Hamid R Farshchi ${ }^{1}{ }^{2}$ (MD, PhD)

- Author Affiliations

1. School of Life Sciences, Queen's Medical Centre, University of Nottingham, Nottingham NG7 2UH, UK. (AM, MAT, IAM, HRF)

2. NovinDiet Clinic, Tehran, Iran. (AM, HRF)

3. Digestive Disease Research Institute, Tehran University of Medical Sciences, Tehran, Iran. (AD, RM)

- To whom correspondence should be addressed. Dr. Hamid R Farshchi, Tel: +9821-22263002, Fax: +9821-22274192, E-mail: hrfarshchi@gmail.com, hamid.farshchi@nottingham.ac.uk

- To whom requests for reprints should be addressed: Name Dr. Ameneh Madjd, Email: $\underline{\text { mbxsama@nottingham.ac.uk }}$

- Running title: Effect of Replacing Diet Beverage by Water in T2DM

- Word counts: 5388 
Page 2 of 29

2

Abbreviations:

- Analysis of variance: ANOVA

- Cognitive behavioral therapy: CBT

- Diet beverages: Bs

- Fasting plasma glucose: FPG

- Glycated hemoglobin: $\mathrm{Hb} \mathrm{A}_{1 \mathrm{C}}$

- Homeostasis model assessment of insulin resistance: HOMA-IR

- Hour: h

- Liter: I

- Mole: mol

- Standard deviation: SD

- Sugar sweetened beverages: SOBs

- Total cholesterol: TC

- Triglyceride: TG

- 2 hour post prandial glucose: $2 \mathrm{hpp}$

- Waist circumference: WC 


\section{Abstract}

4 Aims: To compare the effect of replacing diet beverages (DBs) with water or continuing

5 to drink DBs, in Type 2 diabetes during a 24 week weight loss program. The primary

6 endpoint was the effect of intervention on weight over 24 weeks. The main secondary

7 endpoints included anthropometric measurement, glucose and fat metabolism during

8 the 24 weeks.

9 Methods: 81 Overweight and obese women with type 2 diabetes, who usually

10 consumed DBs in their diet, were asked to either substitute water for DBs or continue

11 drinking DBs five times per week after their lunch for 24 weeks (DBs group), while they

12 were on a weight loss program.

13 Results: Compared with the DBs group, the Water group had a greater decrease in 14 weight (Water: $-6.40 \pm 2.42 \mathrm{~kg}$; DBs: $-5.25 \pm 1.60 \mathrm{~kg} ; P=0.006)$, BMI (Water: $-2.49 \pm$ 150.9 2kg/m²; DBs: $\left.-2.06 \pm 0.62 \mathrm{~kg} / \mathrm{m}^{2} ; P=0.006\right), \mathrm{FPG}($ Water: $-1.63 \pm 0.54 \mathrm{mmol} / \mathrm{l} ; \mathrm{DBs}:-$ $161.29 \pm 0.48 \mathrm{mmol} / \mathrm{l}, \mathrm{P}=0.005)$, fasting Insulin (Water: $-5.71 \pm 2.30 \mathrm{~m} \mathrm{IU} / \mathrm{ml}$; DBs: $-4.16 \pm$ $171.74 \mathrm{~m} \mathrm{IU} / \mathrm{ml}, \mathrm{P}=0.011)$, HOMA IR (Water:-3.20 \pm 1.17 ; DBs: $-2.48 \pm 0.99, \mathrm{P}=003$ ) and 18 2h post prandial glucose (Water: $-1.67 \pm 0.62 \mathrm{mmol} / \mathrm{l}$; DBs: $-1.35 \pm 0.39 \mathrm{mmol} / \mathrm{l}$;

$19 \mathrm{P}=0.027)$ over the 24 weeks. However, there was no significant group * time interaction 20 for waist circumference, lipid profiles and $\mathrm{HbA}_{1 \mathrm{c}}$ within both groups over 24 weeks.

21 Conclusion: Replacement of DBs with water after the main meal in patients with type 2 22 diabetes obese adult women may lead to more weight reduction during a weight loss 23 program. 


\section{Introduction:}

25 There is evidence that the risk of developing type 2 diabetes is associated positively with $\mathrm{BMI}(1,2)$. Obesity also complicates the management of type 2 diabetes by

27 increasing insulin resistance and blood glucose concentrations (3). In contrast, weight reduction is an effective goal for overweight/ obese type 2 diabetes in order to improve

29 glycemic control (4).

In the last decades, the amount of energy consumed in beverages has increased,

31 providing a significant source of daily energy intake (5). Also, to promoting weight gain,

32 a higher intake of sugar sweetened beverages (SSBs) is associated with the

33 development of metabolic syndrome and an increased risk of type 2 diabetes (6). On

34 the other hand, diet beverages (DBs) are of interest as dietary tools which offer sweet

35 taste without energy (7-9).

36 Nutritionists usually advise individuals who wish to lose weight to raise their water

37 consumption $(10,11)$. Conversely, many obese and diabetic patients believe that they

38 can drink DBs during a diet plan without any deleterious effects on their weight and

39 diabetes management (12). A previous review indicated that DBs might be the ideal use

40 of intense sweeteners in the setting of a weight control plan, while they have been

41 shown to be associated with some modest weight loss (13). Thus, it would be expected

42 that Type 2 diabetic patients could consider DBs in order to help them to lose weight

43 and control their blood glucose.

44 Nevertheless, SSBs and DBs intake were associated with a significantly higher risk of

45 type 2 diabetes (14) and a subsequent observational study revealed that consumption

46 of DBs was significantly associated with an increased risk for type 2 diabetes (15). More 
47 experimental study is needed to determine the effect of DBs consumption on the

48 management of diabetes and metabolic syndrome.

49 Recently, we investigated the effect of replacing DBs with water, on promoting weight

50 reduction in obese adults without diabetes who were on a hypo-energetic diet (16). The

51 Water group had a greater decrease in weight and insulin resistance over the 24 weeks

52 of study compared with DBs group. Due to beneficial effects of substitution of DBs with

53 water in overweight/ obese women, it would be interesting to repeat this protocol in

54 those with Type 2 diabetes.

55 Thus, the purpose of this study was to investigate the effects of replacing DB

56 consumption with water during a comprehensive 24-wk weight-loss program on body

57 weight as a primary outcome, along with abdominal adiposity, carbohydrate and lipid

58 metabolism as secondary outcomes, in overweight and obese women with Type 2

59 diabetes.

60 Materials and Methods

61 Study participants

62 Obese female adults with diabetes were selected between April 2015 and June 2015

63 from the participants attending NovinDiet Clinic, Tehran, Iran to lose weight and control

64 diabetes. Inclusion criteria were female, $18-50 \mathrm{y}$ of age, $\mathrm{BMI}=27-35 \mathrm{~kg} / \mathrm{m}^{2}$,

$65 \quad 6.5<\mathrm{Hb}_{\mathrm{A} 1 \mathrm{C}}<7.2$ and only taking Metformin to control their diabetes, self-reported

66 habitual consumers of DBs who were willing to introduce a dietary change to lose

67 weight which might include changing beverage consumption.

68 All participants were required to be nonsmokers, free of established cardiovascular

69 diseases, stroke, liver diseases, kidney diseases, depression, cancer or autoimmune 
70 disease. Subjects included those who were able to keep an adequate 4- day food

71 record and who demonstrated readiness to participate safely in daily physical activity 72 (PA).

73 Exclusion criteria were pregnancy or lactation during the previous 6 months or planned

74 pregnancy in the next 6 months, weight loss $\geq 10 \%$ of body weight within the 6 month

75 before enrollment in the study, taking medication to lower lipids/ cholesterol or that

76 could affect metabolism or change body weight.

77 The study was approved by the Ethical Committee of The Digestive Research Institute,

78 Tehran University of Medical Science. All subjects provided their signed consent prior to

79 study enrollment. This trial was registered at http:/www.clinical trials.gov/ as

$80 \quad$ NCT02412774.

81 Randomization and Intervention

82 The study was a 2-arm, single-blind, randomized clinical trial. Eligible participants were

83 randomly assigned after baseline measures by using a computer-generated random-

84 numbers method by the project coordinator with allocation concealed from the

85 participants and dietitians until randomization was revealed to the study participants at

86 the initial intervention clinic appointment.

87 Eighty-one participants who were eligible for the study were randomly assigned to one

88 of the 2 groups. All had a 2-wk any artificial sweetener products including diet

89 beverages washout period before intervention. The groups were the Water group in

90 which subjects replaced habitual post lunch (main meal) intake of DBs with a glass of

91 water $(250 \mathrm{ml})$ and in the DBs group subjects were instructed to continue to drink DBs

92 once a day $(250 \mathrm{ml})$, after their main meal (lunch) 5 times a week. Both groups were free 
93 to drink water as beverage at other times, but were not allowed to have DBs

94 consumption. In addition, both groups were asked not to drink DBs or water during the

95 lunch meal and also not to add low calorie sweeteners to beverages such as tea or

96 coffee. To control the effects of menstrual cycle on measurements, participants started

97 the study at the same phase of their menstrual cycle. Bi-weekly visits to the dietitian

98 were required in order to promote adherence to the hypo-energetic diet and beverage

99 substitution.

100 Dietary and activity programs

101 NovinDiet Clinic is a private weight loss clinic which uses an integrated approach

102 (dietary, behavioural, exercise and medical treatments). Subjects who participated in

103 this study did not pay clinic fees, were provided the diet beverages for DBs group and

104 water for the Water group over the study. In this study the program was designed to

105 enable weight loss of $7-10 \%$ of starting body weight, at a rate of $0.5-1 \mathrm{~kg} /$ week over 24

106 weeks. The individual diet programs were based on the individual's food diary records,

107 with gradual adjustment to bring their diet in line with the NovinDiet protocol. PA was

108 encouraged; the objective was to gradually increase activity levels to achieve 60

109 minutes of moderate activity on five days/ week. Predominant behavior change

110 strategies applied included stages of change, goal setting, self-monitoring with food

111 diaries and PA $(17,18)$.

112 At bi-weekly sessions, resources were provided as home booklets for each subject to

113 record adherence to the diet protocol. During the intervention period, subjects

114 completed the feedback form regarding their adherence to the diet. Subjects also had 
115 access to a website, weekly internet magazines, and one to one telephone/ online 116 support from a consultant, if needed.

117 Outcomes

118 To assess the effect of replacing DBs with water outcomes were collected at the 119 baseline, 12 weeks and 24 weeks (except height which was taken only at the screening 120 visit).

\section{Anthropometric measurements}

122 Body weight was taken to the nearest $0.1 \mathrm{~kg}$ using a digital calibrated scale (Omron 123 Health Care, Hoofdorp, Netherland), whilst subjects wore light clothing, without shoes. 124 Body height was measured to the nearest $0.1 \mathrm{~cm}$ by using a wall mounted stadiometer 125 (SECA, Hamburg, Germany) while participants were barefoot and in a free-standing 126 position. Waist circumference (WC) was measured with a rigid measuring tape and 127 recorded to the nearest $0.5 \mathrm{~cm}$. WC was measured at the smallest horizontal 128 circumference between the ribs and iliac crest (the natural waist), or, in case of an 129 indeterminable waist narrowing, halfway between the lower rib and the iliac crest (19). 130 BMI was calculated from measured weight in kilogram divided by the square of height in 131 meters.

\section{Blood sample measurements}

133 Blood samples of all subjects were taken after overnight (8-10 h) fasting, between 07:00 134 and 09:00, at baseline, 12 and 24 weeks for biochemical, cellular and hormonal 135 measurements. Fasting blood samples were collected by venipuncture according to a 136 standard protocol. Blood samples were taken while the subjects were in a sitting 137 position, according to the standard protocol, and were centrifuged at $2000 \mathrm{~g}$ at room 
138 temperature within 30-45 min. Antecubital venous blood samples for two-hour 139 postprandial plasma (2hpp) glucose were taken 2 hours after ingesting $75 \mathrm{~g}$ of glucose 140 according to the standard method(20). Fasting plasma glucose (FPG) and $2 \mathrm{hpp}$ plasma 141 glucose levels were measured using the enzymatic colorimetric method. Insulin was 142 measured by using a radioimmunoassay with ${ }^{125}$ I-labeled human insulin and a human 143 insulin antiserum in an immunoradiometric assay (IRMA) (Biosource, Dorest, Belgium) 144 with a gamma-counter system (Gamma I; Genesys). Insulin resistance was evaluated 145 by homeostasis model assessment of insulin resistance (HOMA-IR) (21).

146 Glycated hemoglobin $\left(\mathrm{Hb} \mathrm{A}_{1 \mathrm{C}}\right)$ was measured by a colorimetric method after an initial 147 separation by ion exchange chromatography (Biosystem, Barcelona, Spain).

148 Biochemical analysis of the serum total cholesterol (TC), triglyceride (TG), and high149 density lipoprotein (HDL) cholesterol was carried out on a Selectra E auto analyzer (Vita 150 Laboratory, Netherlands) following standard procedures of the Pars Azmoon diagnostic 151 kits (Iran). The LDL cholesterol was calculated using the Friedewald formula(22).

$152 \mathrm{LDL}$ cholesterol $=\mathrm{TC}-\mathrm{HDL}$ cholesterol $+(\mathrm{TG} \div 2.2)$

153 Self-reported dietary assessment

154 Energy and macronutrient intake at baseline, weeks 11 and week 23 was analyzed by 155 Nutritionist IV software (version 4.1; Hearst).

156 Statistical analyses

157 Baseline values of cardiovascular risk factors (including weight, waist circumference, 158 LDL-C, HDL-c, TC, FPG, TG, fasting insulin, HOMA IR, Hb $\mathrm{A}_{\mathrm{A} 1 \mathrm{C}}$, 2hpp glucose data) were 159 compared between the Water and DBs groups using unpaired $t$-tests. 
160 At baseline, distribution was normal for all variables. All participants who were randomly 161 assigned and completed an initial assessment were included in the final results by using 162 an intention-to-treat analysis. Multiple imputations with the use of linear regression were 163 used to impute missing values from $24 \mathrm{wk}$ and were based on the assumption that data 164 were missing at random.

165 The primary analysis was an intent to treat linear mixed effect, which assessed at 12 166 and 24 weeks. These models, which included time, treatment, a time by group

167 interaction and the respective baseline value as principal explanatory variables for all 81 168 participants. The per-protocol analysis was also done for the outcomes .the results from 169 per-protocol analysis were also similar to those of the intent to treat analysis in direction 170 and significance. Statistical significance was set at $p \leq 0.05$. All data are presented as 171 mean \pm SD unless otherwise stated. Associations between variables were assessed by 172 simple correlational analyses (Pearson's $r$ ). All statistical analyses were performed 173 using SPSS 22.0 for Windows (SPSS Inc., USA).

174 The primary outcome addressed in this study was the difference in body weight loss 175 during the 24 week weight loss program. The power calculation was based on the 176 previous studies $(16,23)(\alpha=0.05$, power $=0.85)$, which were performed based upon 177 expected differences in weight loss between weight loss diet groups $(2.0 \pm 2.5 \mathrm{~kg})$ to 178 determine the targeted final sample size $(n=56)$. Anticipating a dropout rate of $30 \%$ the 179 sample size required was 80.

180 Results

181 Sample characteristics 
182124 patients with type 2 diabetes, who believed that they were eligible and expressed 183 an interest in participating in the study, were evaluated for eligibility by a physician. After 184 evaluation, 81 subjects were recruited and 65 subjects completed the 24 -week 185 intervention (with $80 \%$ retention rate, Figure 1). The remaining 81 subjects gave 186 written consent and then randomly 41 subjects were allocated to the water and 40 to the 187 DBs group. After starting the intervention, a total of 11 subjects dropped out because 188 they did not wish to continue or they moved away from the area. 2 subjects left the 189 study as they became pregnant. The remaining 3 subjects did not give any reason for 190 their withdrawal.

191 At baseline, there were no statistically significant differences in age, physical

192 characteristics or biochemical measurements between the groups or between those 193 who completed or did not complete the study once recruited (Table 1).

194 Body weight

195 As shown in Table-2, there was a significant weight reduction in each group after 24 196 weeks $(P<0.001)$. There was also a significant difference in weight reduction between 197 the two groups after 24 weeks $(P=0.006$, Figure 2).

198 BMI and Waist circumference

199 BMI reduction in each group was in the expected direction with significant effects over 20024 weeks for both groups $(P<0.001)$. However, the decline in $B M I$ was greater in the 201 water group than the DBs group after 24 weeks $(P=0.006)$.

202 In both groups, waist circumference had decreased after 24 weeks of intervention $203(P<0.001)$ with no significant difference in WC effects between the two groups after the 204 intervention $(P=0.833)$. 


\section{Glucose metabolism measurement}

206 Fasting plasma glucose, fasting serum insulin, 2 hour postprandial (2hpp) glucose, $\mathrm{Hb}$

$207 \mathrm{~A}_{1 \mathrm{C}}$ and HOMA-IR all decreased over time in both groups $(P<0.001)$. Also between

208 group differences were significant for all variables (Table 2).

209 There was a significant difference in fasting plasma glucose level changes between the 210 two groups after 24 weeks $(P=0.005)$. In terms of $2 \mathrm{hpp}$, during the 24 weeks of

211 intervention between group changes was significant $(P=0.027)$.

212 There was a significant difference in insulin resistance between the two groups over 24

213 weeks $(P=0.003)$ but no significant improvement in $\mathrm{HbA}_{1 \mathrm{C}}$ in the water group compared 214 with the DBs group over the 24 weeks $(P=0.149)$.

215 Furthermore, Fasting serum insulin concentration decreased significantly over time, with 216 significant differences between the two groups after 24 weeks $(P=0.011)$.

\section{Food intake measurement}

218 At baseline, there was no significant difference in energy intake. Estimated energy 219 intake measurements showed a significant reduction over time in both groups $(\mathrm{P}<$ 2200.001 for time effect). As shown in Table 3, there was a significant group*time 221 interaction for total energy intake over $24 \mathrm{wk}(P=0.005)$.

222 In addition, macronutrient intake measurements showed no significant differences 223 between the 2 groups at baseline. However, there was a greater carbohydrate deficit in 224 the water group than in the DB group during the 24 wk of intervention (group * 225 time interaction, $\mathrm{P}<0.001$, Table 3)

\section{Discussion}


227 The purpose of the present study was to compare the effects of DBs and water

228 consumption after lunch, as a main meal, on weight loss and also characteristics of

229 carbohydrate and lipid metabolism in overweight and obese women with type 2 diabetes 230 attending a weight loss program for 24 weeks. The results of present study showed that 231 drinking water may lead to more weight loss, a greater improvement in fasting plasma 232 glucose, insulin sensitivity, measured by HOMA IR and $2 \mathrm{hpp}$ glucose levels compared 233 with consumption of DBs in women with Type 2 diabetes.

234 To our knowledge, this study was the first randomized controlled trial in women with 235 Type 2 diabetes which has assessed the impact of excluding DBs consumption on 236 weight loss, during a voluntary weight reduction program, for 24 weeks.

237 Weight gain and obesity are strongly related to the increased risk of type 2 diabetes 238 while moderate weight loss improves glycaemic control (1). All of the subjects in our 239 weight loss plan had a significant weight loss. This would have been predicted given the 240 characteristics of the prescribed treatment plan which included energy restriction, PA 241 instruction and regular patient visit and consultation in the clinic. In other rigorous clinic242 based behavioral lifestyle adjustment programs, 5-10\% weight losses have been 243 reported at 6 months (24-26) which is similar to the weight losses reported in our study. 244 These comprehensive weight loss methods are more constantly effective in comparison 245 with others recommending small but theoretically sustainable lifestyle changes that can 246 be made to improve health (27). Furthermore, the present study showed a major 247 reduction in waist circumference and significant improvements in cardio metabolic risk 248 characteristics in both groups over 24 weeks, as would be predictable given the weight 249 loss observed. Although the results indicated a significant effect of replacing DBs with 
250 water on weight loss during 24 weeks, it seems that the 24 weeks of intervention was

251 not enough to reveal any significant effects on waist circumference (WC) as a related

252 metabolic variable. Further longer term studies measuring metabolic effects, including 253 WC, and more accurate assessment of body fat change using DEXA Scanning would 254 be required.Previous intervention studies have attempted to investigate the effects of 255 water and DBs consumption on weight loss with inconsistent results. In a recent study, 256 the effects of either water or DBs consumption in comparison to SSBs, without any 257 hypoenergetic diet, and only having group behavioural counselling to promote 258 adherence to beverage substitution were compared(28). The authors failed to find any 259 significant differences in weight loss between water and DBs. In another study(29), 260 drinking water and diet beverage was compared in subjects undergoing cognitive 261 behavior therapy only, with no specific dietary restrictions. The result of this study 262 showed a greater impact on weight loss with DBs compared with water. On the other 263 hand, in a study by Dennis et al. (30), subjects who were randomly assigned to drink 264 pre-meal water lost about $2 \mathrm{~kg}$ more weight than subjects on an hypoenergetic diet 265 alone. It should be noted that the protocol of the last study (30) was not similar to our 266 study in that subjects in both groups had either water or DBs after their meal rather than 267 before the meal, which is more representative of normal behaviour in this group. 268 Furthermore, none of these studies involved obese or overweight subjects with Type 2 269 diabetes. Following our recent study (16) indicating the beneficial effects of replacing 270 diet beverages with water on weight loss and insulin sensitivity of obese and overweight 271 adults, our goal was to investigate whether these effects may be seen in women with 272 Type 2 diabetes. 
273 In our current study, participants drinking water after their lunch over 24 weeks lost 1.16

$274 \mathrm{~kg}$ more than those in the DBs group, which is in agreement with the result of our

275 previous study(16) where the overweight/ obese but otherwise healthy women in the

276 water group lost $1.2 \mathrm{~kg}$ more than the DBs group. In contrast, our results are

277 inconsistent with other studies which indicated either no significant change in effects on

278 weight loss between water and DBs (28) or reported greater impact on weight loss with

279 DBs compared with water(29). Nevertheless, it should be mentioned that these studies

280 had different experimental designs, for example not including any weight loss plan(28)

281 or have cognitive behavioral therapy alone for weight loss during a shorter period of 12

282 weeks(29). Also, the volume of beverage, the time of drinking and the type of

283 participants were different in these studies.

284 The results of our latest study may have arisen because the effect of replacement of

285 DBs with water may lead to better adherence to the weight loss diet in the Water group.

286 It has been hypothesized that artificial sweeteners may raise the hedonic desire for

287 sweetened and more energy dense foods (31-33). Also in our current study, the effect

288 of replacement of DBs with water on weight loss reflected better adherence to the

289 weight-loss diet in the water group. The greater reduction in energy intake in water

290 group compared with DBs group resulted in more weight loss in this group than DBs

291 group. Moreover, more reduction of carbohydrate consumption in the water group than

292 in the DBs group might support greater weight loss in the water group. However, in

293 order to elucidate the mechanism that might explain the better weight loss in the Water

294 group compared with the DBs group, longer term studies are required. 
295 Like our study on healthy overweight/obese women (16), our present study in women

296 with Type 2 diabetes revealed a better improvement in fasting insulin sensitivity, (

297 HOMA IR), in the Water group over the 24 weeks. there was also a beneficial impact of 298 on fasting glucose and $\mathrm{Hb}_{\mathrm{A} 1 \mathrm{C}}$ in the Water group, although our previous study in 299 women without diabetes(16) did not show any effects on these carbohydrate 300 metabolism characteristics. But these outcomes seen on diabetic patients were 301 consistent with the results of the recent epidemiological study indicating daily diet 302 beverage consumption was associated with impaired glucose control(32).

303 These results may have clinical implications, showing that if overweight/ obese people 304 with Type2 diabetes use a weight loss plan, they may have better improvements in 305 glycemic characteristics and weight loss if they drink water instead of DBs. These 306 findings would reinforce the recommendations given in popular weight loss programs 307 that the obese and overweight patients who are keen to lose weight should increase 308 their water intake $(10,11)$. On the other hand, most obese people consider that they 309 can drink diet beverages during a low- energy diet without any harmful effects on their 310 weight management, and whilst they do still lose weight, the magnitude of the weight 311 loss may be greater if they avoid DBs completely. Whilst the present study is consistent 312 with the current guideline for increasing water consumption for better diabetes control, 313 our results do not entirely support the recommendations indicating no deleterious 314 effects of diet beverage on diabetes control(34). Since the consumption of diet soda is 315 higher among people with diabetes than those without (35), the potential implications of 316 studies such as ours needs further investigation. 
317 The main strength of this study is that it was a randomized, outpatient clinical trial, whilst

318 participants were selected from participants wished to lose weight and control their

319 blood sugar and included middle-aged overweight and obese women who were able to 320 comply with weight-loss plan; hence, they demonstrated that they were motivated to 321 adhere to the weight-loss diet protocol (36). Thirdly Subjects who participated in this 322 study did not pay clinic fees and were provided the diet beverages for DBs group and 323 water for the Water group which were incentive for regular by-weekly visits with the 324 dietitian when compliance could be encouraged in both groups.

325 On the other hand, there are some limitations. First of all, even though the sample size 326 providing sufficient power to distinguish statistically significant effects in the key 327 outcome variables, the sample was not representative of the general population, 328 mainly as it did not include men. In addition, due to the possible effects of the time of 329 the beverage consumption, we only asked the participants to drink either water or diet 330 beverages after the lunch in order to cover this confounding factor. Also we did not 331 record the fluid intake of participants as it may influence satiety. Moreover the energy 332 expenditure was not verified which would affect weight loss. Lastly, although our 333 weekly follow up by phone call and fortnight clinic visit to measure dietary compliance of 334 the subjects, the present study

335 only relied upon subjective report of storing and consuming the water and DBs which is 336 not as accurate as objective methods for measuring their compliance.

337 In conclusion, replacing DBs with water consumption would appear to impact 338 beneficially on weight loss, BMI, FPG and insulin sensitivity in overweight and obese 
339 women with Type 2 diabetes following a weight loss diet. However, longer term studies 340 are essential to see what would happen in long term in such patients.

341 


\section{Author contributions:}

343 Experiments in this study were conducted in NovinDiet Clinic, Tehran. AM: contributed

344 to the initial study design, study protocol setup, data collection, data analysis, and 345 writing of the first draft of the manuscript; HRF: designed the research, conducted the 346 research, contribution to data interpretation, revision of the manuscript and provided 347 medical supervision; MAT, IAM: refined the study design and contributed to data 348 interpretation and redrafting of the manuscript.RM and AD: provided advice and 349 consultation for the study design, conducted the research. All authors read and 350 approved the final manuscript. HRF is the guarantor of this work and, as such, had full 351 access to all the data in the study and takes responsibility for the integrity of the data 352 and the accuracy of the data analysis.

\section{Acknowledgements}

354 The authors thank the participants and staff of NovinDiet Clinic for their contribution to 355 this study; Mansoureh Pahlevani, Zeynab Zolfaghari, Rahil Ahmadi and Ziba

356 Hooshmand for their assistance in data collection and Dr. Masoud Solaymani and Dr.

357 Leila Janani, Iran University of Medical Sciences, for statistical consultation. Thanks

358 also go to Dr. Kourosh Asadi at Jaam e Jam Laboratory for the analysis of blood 359 samples.

360 Funding: This study was supported by The school of Life Sciences, The University of 361 Nottingham, Nottingham, UK and The Digestive Disease Research Institute (DDRI), 362 affiliated to Tehran University of Medical Sciences (TUMS).

363 Duality of Interest: No potential conflicts of interest relevant to this article were 364 reported. 


\section{Reference}

1. Klein S, Sheard NF, Pi-Sunyer X, et al. Weight Management Through Lifestyle

Modification for the Prevention and Management of Type 2 Diabetes: Rationale and Strategies:

A statement of the American Diabetes Association, the North American Association for the

Study of Obesity, and the American Society for Clinical Nutrition. Diabetes Care.

$2004 ; 27(8): 2067-73$.

2. Colditz GA WW, Stampfer MJ, London SJ, Segal MR, Speizer FE. Patterns of weight change and their relation to diet in a cohort of healthy women. Am J Clin Nutr 1990;51:1100-5.

3. Maggio CA, Pi-Sunyer FX. The prevention and treatment of obesity. Diabetes care. $1997 ; 20(11): 1744$.

4. Franz MJ, Bantle JP, Beebe CA, et al. Evidence-Based Nutrition Principles and Recommendations for the Treatment and Prevention of Diabetes and Related Complications. Diabetes Care. 2002;25(1):148-98.

5. Duffey KJ PB. Shifts in patterns and consumption of beverages between 1965 and 2002. . Obesity 2007;15:2739-47.

6. Malik VS PB, Bray GA, Després JP, Willett WC, Hu FB. Sugar-sweetened beverages and risk of metabolic syndrome and type 2 diabetes: a meta-analysis. Diabetes Care 2010;33:247783.

7. Benton D. Can artificial sweeteners help control body weight and prevent obesity? Nutrition Research Reviews. 2005;18(01):63-76.

8. Bellisle F, Drewnowski A. Intense sweeteners, energy intake and the control of body weight. European Journal of Clinical Nutrition. 2007;61(6):691-700. 
9. Mattes RD, Popkin BM. Nonnutritive sweetener consumption in humans: effects on appetite and food intake and their putative mechanisms. The American journal of clinical nutrition. 2009;89(1):1-14.

10. Atkins R. Atkins' NEW Diet Revolution. New York: Avon Books; 1992.

11. Sears B LW. The Zone. New York: Harper Collins; 1995.

12. Morris DH, Cuneo $P$, Stuart MJ, et al. High-intensity sweetener, energy and nutrient intakes of overweight women and men participating in a weight-loss program. Nutrition Research. 1993;13(2):123-32.

13. De la Hunty A, Gibson S, Ashwell M. A review of the effectiveness of aspartame in helping with weight control. Nutrition Bulletin. 2006;31(2):115-28.

14. de Koning LMV, Rimm EB, Willett WC, Hu FB. Sugar-sweetened and artificially sweetened beverage consumption and risk of type 2 diabetes in men. Am J Clin Nutr. 2011(93):1321-7.

15. Sakurai M, Nakamura K, Miura K, et al. Sugar-sweetened beverage and diet soda consumption and the 7-year risk for type 2 diabetes mellitus in middle-aged Japanese men. European journal of nutrition. 2014;53(1):251-8.

16. Madjd A, Taylor MA, Delavari A, Malekzadeh R, Macdonald IA, Farshchi HR. Effects on weight loss in adults of replacing diet beverages with water during a hypoenergetic diet: a randomized, 24-wk clinical trial. The American Journal of Clinical Nutrition. 2015;102(6):130512.

17. Panel NOEIE. Clinical guidelines on the identification, evaluation, and treatment of overweight and obesity in adults. 1998. 
18. Initiative NOE, Heart N, Obesity NAAftSo, Identification EPot, Overweight To, Adults Oi. The practical guide: identification, evaluation, and treatment of overweight and obesity in adults: National Heart, Lung, and Blood Institute; 2002.

19. Mason C, Katzmarzyk PT. Effect of the Site of Measurement of Waist Circumference on the Prevalence of the Metabolic Syndrome. The American journal of cardiology. 2009;103(12):1716-20.

20. Association AD. Diagnosis and Classification of Diabetes Mellitus. Diabetes Care. 2014;37(Supplement 1):S81-S90.

21. Matthews DR HJ, Rudenski AS, Naylor BA, Treacher DF, Turner RC. Homeostasis model assessment: insulin resistance and beta-cell function from fasting plasma glucose and insulin concentrations in man. Diabetologia 1985;28(7):412-19.

22. Friedewald WT, Levy RI, Fredrickson DS. Estimation of the Concentration of Low-Density Lipoprotein Cholesterol in Plasma, Without Use of the Preparative Ultracentrifuge. Clinical Chemistry. 1972;18(6):499-502.

23. Dennis EA, Dengo AL, Comber DL, et al. Water Consumption Increases Weight Loss During a Hypocaloric Diet Intervention in Middle-aged and Older Adults. Obesity. 2010;18(2):300-7.

24. Look AHEAD Research Group. Long term effects of a lifestyle intervention on weight and cardiovascular risk factors in individuals with type 2 diabetes: four year results of the Look AHEAD trial. Archives of internal medicine. 2010;170(17):1566.

25. Appel LJ, Champagne CM, Harsha DW, Cooper LS, E O. Die Auswirkungen von «Lifestyle»-Interventionen auf den Blutdruck. JAMA. 2003;289:2083-93. 
26. Diabetes Prevention Program Research Group. Reduction in the incidence of type 2 diabetes with lifestyle intervention or metformin. The New England journal of medicine. 2002;346(6):393.

27. Stroebele N, de Castro JM, Stuht J, Catenacci V, Wyatt HR, Hill JO. A small-changes approach reduces energy intake in free-living humans. Journal of the American College of Nutrition. 2009;28(1):63-8.

28. Deborah F Tate GT-M, Elizabeth Lyons, June Stevens, et al. Replacing caloric beverages with water or diet beverages for weight loss in adults: main results of the Choose Healthy Options Consciously Everyday (CHOICE) randomized clinical trial. Am J Clin Nutr. 2012;95(3): 555-63. .

29. Peters JC, Wyatt HR, Foster GD, et al. The effects of water and non-nutritive sweetened beverages on weight loss during a 12-week weight loss treatment program. Obesity. $2014 ; 22(6): 1415-21$.

30. Dennis EA, Comber DL, Flack KD, et al. Water consumption increases weight loss during a hypocaloric diet intervention in middle-aged and older adults. . Obesity (Silver Spring) 2010;18:300-7.

31. Blundell JE HA. Paradoxical effects of an intense sweetener (aspartame) on appetite. Lancet 1986;1:1092-3.

32. Nettleton JA1 LP, Wang Y, Lima JA, Michos ED, Jacobs DR Jr. Diet soda intake and risk of incident metabolic syndrome and type 2 diabetes in the Multi-Ethnic Study of Atherosclerosis (MESA). Diabetes Care. 2009;32(4):688-94. 
33. Rogers PJ BJ. Separating the actions of sweetness and calories: effects of saccharin and carbohydrates on hunger and food intake in human subjects. Physiol Behav 1989;45:1093-9.

34. (ADA) ADA. What Can I Drink? 2014 [updated 2014]. Available from:

http://www.diabetes.org/food-and-fitness/food/what-can-i-eat/making-healthy-foodchoices/what-can-i-drink.html.

35. Mackenzie T, Brooks B, O'Connor G. Beverage Intake, Diabetes, and Glucose Control of Adults in America. Annals of Epidemiology.16(9):688-91.

36. Kraschnewski JL, Boan J, Esposito J, , et al. Long-term weight loss maintenance in the United States. International journal of obesity (2005). 2010;34(11):1644-54. 
Table 1. Baseline characteristics before the intervention*

\begin{tabular}{lcc}
\hline \hline & water group(n=41) & DBs Group(n=40) \\
Age (y) & $34.15(6.99)$ & $35.45(7.45)$ \\
Body wt (kg) & $83.92(4.42)$ & $84.70(7.43)$ \\
Height (cm) & $159.83(2.83)$ & $159.65(3.08)$ \\
BMI (kg/m²) & $32.86(1.67)$ & $33.19(2.25)$ \\
WC (cm) & $103(5)$ & $102(7)$ \\
Married & $78 \%$ & $82 \%$ \\
TC ( mmol/l) & $4.78(0.43)$ & $4.75(0.37)$ \\
HDL-C (mmol/l) & $1.13(0.19)$ & $1.13(0.17)$ \\
LDL-C (mmol/l) & $2.73(0.51)$ & $2.71(0.38)$ \\
TG (mmol/l) & $2.02(0.27)$ & $1.97(0.25)$ \\
FPG (mmol/l) & $8.49(0.90)$ & $8.48(1.03)$ \\
2hppG (mmol/l) & $8.82(1.14)$ & $8.76(1.22)$ \\
HA1C (\%) & $6.97(0.77)$ & $6.95(0.20)$ \\
Insulin ( mU/l) & $19.99(4.07)$ & $19.84(4.07)$ \\
HOMA-IR & $7.59(1.93)$ & $7.50(1.89)$ \\
\hline \hline
\end{tabular}

* Group difference, $\mathrm{P}>0.05$.

Data are presented as mean ( SD)

Diet beverages: DBs, Waist circumference: WC, Total cholesterol: TC, Triglyceride: TG, Fasting plasma Glucose: FPG, 2 hour post prandial glucose: Glycated haemoglobin: HA1C, Homeostasis model assessment of insulin resistance: HOMA-IR 
Table 2. Anthropometric and blood measurement characteristics in Water and DBs Groups at baselien,12 and 24-week interventions*

\begin{tabular}{|c|c|c|c|c|c|c|c|}
\hline & \multicolumn{3}{|c|}{ Water Group(n=41) } & \multicolumn{3}{|c|}{ DBs Group $(n=40)$} & \multirow[t]{2}{*}{$\underline{P}$ for time $\times$ group } \\
\hline & Baseline & week 12 & week 24 & Baseline & week 12 & week 24 & \\
\hline Weight, kg & $83.92(4.42)$ & $79.96(4.86)$ & $77.52(4.95)$ & $84.70(7.43)$ & $81.25(7.03)$ & $79.45(6.99)$ & 0.006 \\
\hline BMI, kg/m² & $32.86(1.67)$ & $31.32(2)$ & $30.36(2.06)$ & $33.19(2.25)$ & $31.84(2.13)$ & $31.14(2.12)$ & 0.006 \\
\hline $\mathrm{WC}, \mathrm{cm}^{\ddagger}$ & $103(5)$ & $99(7)$ & $97(7)$ & $103(7)$ & $99(7)$ & $97(6)$ & 0.832 \\
\hline TC, $\mathrm{mmol} / \mathrm{I}^{\ddagger}$ & $4.78(0.43)$ & $4.52(0.45)$ & $4.29(0.41)$ & $4.75(0.37)$ & $4.49(0.37)$ & $4.31(0.33)$ & 0.119 \\
\hline HDL-C, mmol/l ${ }^{\ddagger}$ & $1.13(0.19)$ & $1.23(0.18)$ & $1.33(0.17)$ & $1.13(0.17)$ & $1.25(0.18)$ & $1.33(0.16)$ & 0.319 \\
\hline LDL-C, mmol// ${ }^{\ddagger}$ & $2.73(0.51)$ & $2.49(0.49)$ & $2.22(0.46)$ & $2.71(0.38)$ & $2.44(0.40)$ & $2.24(0.35)$ & 0.07 \\
\hline TG, mmol/l $\ddagger$ & $2.02(0.27)$ & $1.77(0.28)$ & $1.63(0.27)$ & $1.97(0.25)$ & $1.75(0.24)$ & $1.62(0.19)$ & 0.639 \\
\hline FPG, mmol/l & $8.49(0.90)$ & $7.76(0.82)$ & $6.86(0.77)$ & $8.48(1.03)$ & $7.85(0.96)$ & $7.19(0.81)$ & 0.005 \\
\hline 2hpp, mmol/l & $8.82(1.14)$ & $7.91(0.87)$ & $7.15(0.70)$ & $8.76(1.22)$ & $8.03(1.06)$ & $7.40(1.01)$ & 0.027 \\
\hline $\mathrm{Hb}$ A1C, $\% \mp$ & $6.97(0.77)$ & $6.16(0.99)$ & $5.80(0.82)$ & $6.95(0.20)$ & $6.81(0.17)$ & $6.53(0.16)$ & 0.149 \\
\hline Insulin, m U/I & $19.99(4.07)$ & $16.75(4.03)$ & $14.27(3.81)$ & $19.84(4.07)$ & $17.36(3.43)$ & $17.36(3.43)$ & 0.011 \\
\hline HOMA-IR & $7.59(1.93)$ & $5.80(1.62)$ & 4.39 ( 1.37$)$ & $7.50(1.89)$ & 6.05 (1.39) & $5.01(1.20)$ & 0.003 \\
\hline
\end{tabular}

* Data are presented as mean (SD) for the 81 participants

$\dagger P$ values are for Water relative to DBs group (time $\times$ Group interaction) by a linear mixed model analysis with repeated measures. $\ddagger$ Significant main effect of time, $\mathrm{P}<0.001$

Diet beverages: DBs, Waist circumference :WC, Total cholesterol: TC,Triglyceride: TG,Fasting plasma glucose: FPG

Glycated hemoglobin: Hb A1C, 2 hour post prandial: 2hpp,Homeostasis model assessment of insulin resistance: HOMA-IR 
Table 3. Self-reported dietary intake in Water and DBs Groups before and after the 24-week interventions*

\begin{tabular}{|c|c|c|c|c|c|}
\hline \multirow[t]{2}{*}{ Intake } & \multicolumn{2}{|c|}{ Water $\operatorname{Group}(n=41)$} & \multicolumn{2}{|c|}{ DBs $\operatorname{Group}(n=40)$} & \multirow[t]{2}{*}{$\underline{\text { P for time } \times \text { group }^{\dagger}}$} \\
\hline & Baseline & week 24 & Baseline & week 24 & \\
\hline Total Energy (kcal) & $2202(173)$ & $1785(146)$ & $2157(275)$ & $1827(302)$ & 0.005 \\
\hline Protein (g) & $81.2(8.7)$ & $79.6(9.4)$ & $80.4(13.9)$ & $80(15.6)$ & 0.240 \\
\hline Protein (\%) & $14.8(2)$ & $17.9(1.6)$ & $14.9(2.1)$ & $17.5(1.8)$ & \\
\hline Fat $(g)$ & $86.2(11.7)$ & $63.8(8.1)$ & $82.6(16.6)$ & $61.1(12)$ & 0.675 \\
\hline Fat (\%) & $35.1(2.8)$ & $32.1(2.3)$ & $34.4(4.3)$ & $30.1(2.4)$ & \\
\hline Carbohydrate (g) & $275.4(20.9)$ & $223(17.6)$ & $273.1(34.5)$ & $239.1(38.6)$ & $<0.001$ \\
\hline Carbohydrate (\%) & $50.1(1.7)$ & $50(2.5)$ & $50.7(3.2)$ & $52.4(2.4)$ & \\
\hline Fiber(g) & $20.7(5.3)$ & $22.2(5.3)$ & $20.8(3.1)$ & $22.2(3.2)$ & 0.280 \\
\hline
\end{tabular}

* Data are presented as mean (SD) for the 81 participants

$\dagger P$ values are for Water relative to DBs group (time $\times$ Group interaction) by repeated-measures two way ANOVA 

28 Subject= Did not 2 grish to continue 30

37 Subject $=$ 3Become pregnant 33

34 Subject= 35 3 Bloved away 37 38 Subject $=$ 3 Quithout reason 40

41

fe 43 43 to 45 continue 46 471 Subject= 48Moved away

4
5
5
5
5
5
5
5
5
5
5
6

Visit 1: Medical History, Beck's depression questionnaire, 4day food Record, physical activity readiness questionnaire

Visit 2: fasting blood draw, 4-day food record collection
Hypo caloric diet+ drink water after lunch $(n=41)$

"Water group"

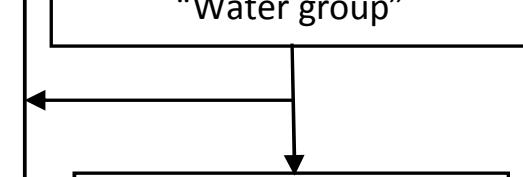

Attended 12 -week assessment $(n=35)$

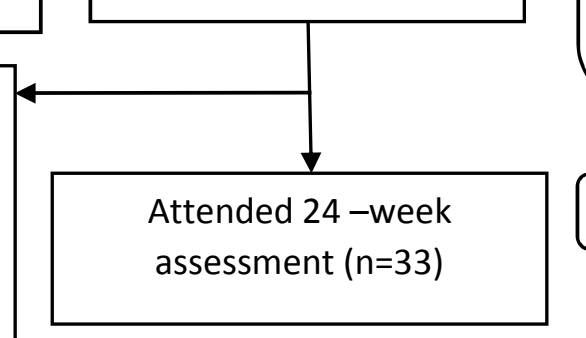
body weight check

Food record collection at week 12 and 24 week
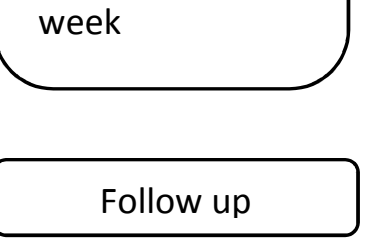

Attended 24 -week assessment $(n=32)$
Excluded $(n=43)$

Beck's depression result $\quad(n=4)$

$\mathrm{BMI}$ out of range

$(n=11)$

Age

$(n=5)$

Disease

$(n=6)$

Fill FD inadequately

$(n=3)$

Others

$(n=14)$

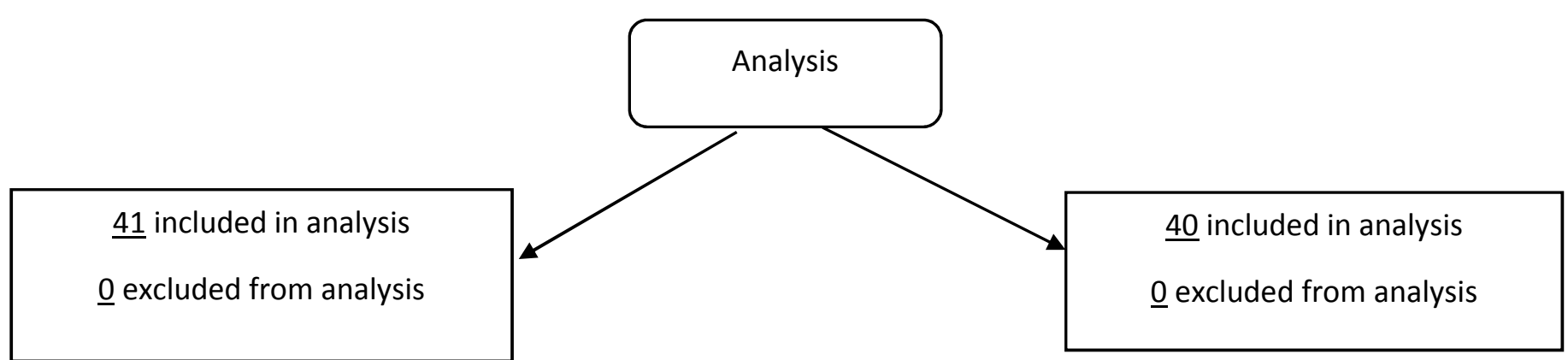


86.00

Figure 2 Mean (SE) weight at baseline, 12 and 24 wk of energy restriction with either drinking water (Water; $n=41$ ) or diet beverages (DBs; $n=40$ ) in all participants, regardless of attrition. $P$ $<0.001$ for the main effect of time. There was also a significant difference in weight reduction between the two groups after 24 weeks $(P=0.006)$, based on linear mixed effects models. 